\title{
Formation of Solid Thorium Monoxide at Near-Ambient Conditions as Observed by Neutron Reflectometry and Interpreted by Screened Hybrid Functional Calculations
}

\author{
Heming $\mathrm{He}^{1, \dagger}$, Jaroslaw Majewski ${ }^{2,5 *}$, David D. Allred ${ }^{3 *}$, Peng Wang ${ }^{2, \ddagger}$, Xiaodong Wen ${ }^{4, \S}$, and Kirk \\ D. Rector ${ }^{1}$.
}
${ }^{1}$ Chemistry Division, Los Alamos National Laboratory, Los Alamos, NM 87545, USA; ${ }^{2}$ MPA/CINT/Lujan Neutron Scattering Center, Los Alamos National Laboratory, Los Alamos, NM 87545, USA; ${ }^{3}$ Department of Physics and Astronomy, Brigham Young University Provo, UT 84602, USA, ${ }^{4}$ Theoretical Division, Los Alamos National Laboratory Los Alamos, NM 87545, USA; ${ }^{5}$ Departement of Chemical Engineering, University of California Davis, Davis, CA, 95616, USA.

KEYWORDS: thorium monoxide, thorium dioxide, neutron reflectometry, nuclear fuel, thin film, oxidation, DFT.

\begin{abstract}
Oxidation of a $\sim 1000 \AA$ sputter-deposited thorium thin film at $150^{\circ} \mathrm{C}$ in $100 \mathrm{ppm}$ of flowing oxygen in argon produces the long-sought solid form of thorium monoxide. Changes in the scattering length density (SLD) distribution in the film over the 700-min experiment measured by in-situ, dynamic neutron reflectometry (NR) shows the densities, compositions and thickness of the various thorium oxides layers formed. Screened, hybrid density-functional theory calculations of potential thorium oxides aid interpretation, providing atomic-level picture and energetics for understanding oxygen migration. NR provided evidence of the formation of substoichiometric thorium oxide, $\mathrm{ThO}_{y}(y<1)$ at the interface between the unreacted thorium metal and its dioxide overcoat which grows inward, consuming the thorium at a rate of $2.1 \AA$ Àmin while y increases until reaching $1: 1$ oxygen-tothorium. Its presence indicates that kinetically-favored solid-phase $\mathrm{ThO}$ can be preferentially generated as a majority phase under the thermodynamically-favored $\mathrm{ThO}_{2}$ top layer at conditions close to ambient.
\end{abstract}

\section{Introduction}

Thorium is one of only two actinides with commercial applications independent of its radioactive nature. Thorium is used to impart high strength and creep resistance in magnesium alloys. [1] [2] It also has utility as an oxide as a catalyst [3] and in high-quality lenses for cameras and scientific instruments. [4]. The phase diagram for thorium and oxygen shows that only one oxide phase is thermodynamically present at ambient pressure: thorium dioxide. [5] $\mathrm{ThO}_{2}$ has one of the highest melting point $\left(3300{ }^{\circ} \mathrm{C}\right)$ of all oxides [6] and thus used in heat-resistant ceramics [3] and in mantles of portable gas lights. The availability of $5 f$ orbitals diffuse enough to be involved in molecular bonding enables unusual chemical compounds. [7] Thorium atoms can also bond to more atoms at one time than any other element. For instance, in the compound thorium aminodiboranate, thorium has a coordination number of fifteen. [7]

The most important future application of thorium may be as an advanced nuclear fuel. Thorium ( $\left.{ }^{232} \mathrm{Th}\right)$ is not itself fissile and so is not the fissile fuel in a thermal neutron reactor. However, it is 'fertile' and upon absorbing a neutron will transmute to ${ }^{233} \mathrm{U}$, which is an excellent fissile fuel and has considerable advantages including safety and proliferation resistance. [8] [9] Current research in nuclear power generation is aimed at reduction of Pu and minor actinides in the spent light water reactor's fuel stockpiles. [10] Considerable research is underway to evaluate the suitability of Th as a nuclear fuel. However, both simplest forms considered- the metal or the dioxide-have significant disadvantages. [11] For example, because thoria's inertness, it is difficult to dissolve spent $\mathrm{ThO}_{2}$-based fuels in nitric acid. [8] Therefore, production and characterization of new thorium-based materials for nuclear fuels is of great importance.

Could a metastable, solid ThO be such a material? The gas-phase, diatomic molecule, ThO, is long known. [12] It can be formed during the vaporization of $\mathrm{ThO}_{2}$. [13] Recently, ThO has been produced through laser ablation of Th metal in the presence of oxygen, [14] [15] [16] and has been characterized in both the gas phase and in a cryogenic matrix. [14] [17] It is being studied in the search for a permanent electron electric dipole moment (eEDM), an important fundamental modern physics problem. [18] [19] Interestingly, recent theoretical work suggests that solid ThO is stable under high-pressure conditions. [20] Nevertheless, the clear demonstration of solid ThO as a dominant phase at ambient conditions has not been observed, though it has been long-sought. [21] [22]

Evidence for the production under mild heating of relatively stable, solid-phase ThO is here demonstrated. It is further shown that ThO can constitute the largest volume of the thorium oxide phases present. The detection method is neutron reflectometry (NR). NR is known as a noncontact, nondestructive, A-resolution analytical technique for characterizing chemical speciation of thin films, including nuclear materials. [23] This paper also describes how the NR method was extended as a time-resolved (i.e., dynamic), in situ tool to identify the presence, stoichiometry and growth rate of subsurface layers under controlled oxidation conditions. Specifically, NR was employed to understand the oxidation of thorium in $\sim 100 \mathrm{ppm}$ of oxygen at $150^{\circ} \mathrm{C}$. Both the growth rate of the $\mathrm{ThO}_{2}$ surface layer and of a substoichiometric $\mathrm{ThO}_{w}$ 
$(w \leq 1)$ layer formed between the $\mathrm{ThO}_{2}$ top layer and the unreacted thorium metal beneath it were measured. To aid in interpretation of the experimental observations screened hybrid-functional calculations were performed on various hypothetical thorium-oxygen structures. This work, also reported herein, provides evidence that a stable ThO layer can formed for kinetic, rather than, thermodynamic reasons.

\section{Experimental and Computational Methodologies}

\section{Thorium Thin-Film Deposition}

A nominal 1000 Å thick thorium film was deposited on a $\sim 7.62-\mathrm{cm}$ diameter, $1-\mathrm{cm}$ thick crystalline quartz substrate for the neutron reflectometry experiments by DC-magnetron sputtering. Special care was taken to achieve low impurity content and small thickness variation in the sputtered film.

Prior to the deposition the cryopumped, high-vacuum deposition chamber achieved a base pressure $<2 \mathrm{x}^{-4} \mathrm{~Pa}^{-1}$ determined by an ion gauge. The sputter working gas was high purity argon. System pressure could be set by adjusting its flow rate. The system pressure during sputtering was a compromise to achieve stable plasma which requires higher pressure and increasing the mean free path of the sputtered atoms by lowering the pressure. Low pressure promotes good film adhesion to the substrate and high film density. The pressure was adjusted to be $\sim 0.35 \mathrm{~Pa}$ as determined using a "o.1 torr" Baratron (capacitance manometer). In the DC- magnetron sputtering system, a Meivac MAK sputter source with a $10-\mathrm{cm}$ diameter thorium target (raw material from Nuclear Fuel Services) was used in the "sputter-up" configuration. The target-to-substrate distance was adjustable from about $-2 \mathrm{~cm}$ (that is, the target could be adjusted to be above the level of the substrate) to $15 \mathrm{~cm}$. Prior to film deposition the thorium target was sputtered in the $-2 \mathrm{~cm}$ position to prevent sputtered atoms from reacting the surface of the substrate. This presputtering for $\sim 5-7$ min removes the native oxide layer and ensures that the thorium deposited on the substrate is as oxygen-free as possible. Prior studies had shown that no atoms are deposited during this cleaning operation. Since the native thorium oxide is an insulator its removal from the surface of the target can be therefore detected as the gradual decrease and plateauing of the magnitude of the target voltage.

Thickness uniformity and surface smoothness are essential for the NR measurements. Three factors contributed to thickness uniformity. First, a planetary system was employed. That is, the substrate was spun around its central axis as the sample tray was slowly rotated over the target at $15 \%$ for 20 revolutions. In addition, a large substrate-target distance (10 $\mathrm{cm}$ ) was chosen. Lastly, a large target was employed. For the same target-substrate distance, sputtering from a large target is inherently capable of producing better uniformity. This is because the atoms emerge from a distributed source. Due to the distribution of the magnetic field behind the target in the MAK sputter gun most of the sputtered atoms emerge from a circular race track about $6 \mathrm{~cm}$ in diameter on the surface of the thorium target. The magnets confine electrons in the plasma to a region near the surface of the target creating high density plasma at low pressures. Previous studies had shown that this sputtering configuration produces thickness variations of less than $5 \%$ over a diameter of $5 \mathrm{~cm}$ on the substrate.

\section{Oxidation Experiments and In-Situ Neutron Reflectometry Measurements}

Time-resolved in-situ NR measurements were performed on the thorium thin film as it was slowly oxidized in a sealed, ceramic-coated, custom-built chamber with controlled oxygen content. The chamber was equipped with low neutron absorbing quartz windows, a two-channel oxygen sensor (Servomex, Inc.), and a gas handling system. Controlled oxidizing environments were created by mixing high-purity $\mathrm{Ar}$ and $\mathrm{O}_{2}$ gases at the desired concentrations and heating the substrate (up to $150 \pm 3^{\circ} \mathrm{C}$ ). The concentration of $\mathrm{O}_{2}$ was measured both upstream and downstream from the reaction chamber.

Once the sample was inside the reaction chamber it was purged using $100 \%$ Ar gas. After the $\mathrm{O}_{2}$ concentration achieved a stable value below $5 \mathrm{ppm}$, indicating that the reaction chamber was leak-tight, the sample was heated to $150^{\circ} \mathrm{C}$. At this point a NR spectrum (Run "o") was collected. Afterwards, $\mathrm{O}_{2}$ was added to the system until a concentration of $\sim 100 \mathrm{ppm}$ $\mathrm{O}_{2}$ in Ar was reached and the subsequent NR spectra were collected. The time-of-flight (TOF) Surface Profile Analysis Reflectometer (SPEAR) instrument at the Los Alamos National Laboratory Lujan Neutron Scattering Center was used to obtain NR data. [24] The neutron beam is produced from a spallation source and, after moderation by liquid $\mathrm{H}_{2}$, is directed onto the sample at a very low angle while the specular reflection is recorded by a TOF position-sensitive detector. During a NR experiment, a collimated beam of neutrons is directed on a planar substrate at a small angle, $\theta$ (usually $0.5-4^{\circ}$ ), and the ratio of the numbers of the elastically scattered to the incident neutrons is measured in the specular geometry. This ratio is defined as the reflectivity or reflectance, $R(Q)$, and is reported as a function of the magnitude of neutron momentum transfer vector and is denoted as $Q$,

$$
|\vec{Q}|=\frac{4 \pi \sin (\theta)}{\lambda}
$$

where $\lambda$ is the neutron wavelength. The momentum transfer vector $\vec{Q}$ is defined as the difference between outgoing and incoming neutron's wavevectors. For the specular geometry, it is perpendicular to the sample surface. The wavelength of a neutron is determined from its velocity $v$ by measuring the time it takes to travel the length of the instrument and by 
using de Broglie expression, $=\frac{h}{m_{n} v}$, where $m_{n}$ is the neutron mass.

In the present TOF NR measurements, the neutron wavelength range varied from 4.5 to $16 \AA$. For the data presented in this manuscript NR for the entire $Q$-range was covered by measurements performed at 4 different angles of incidence

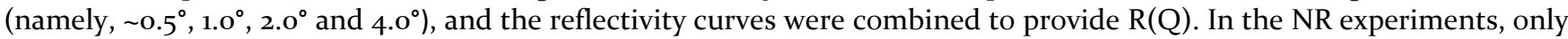
specular scattering was analyzed, although the "off-specular" signal was also recorded. The "off-specular" data provides the neutron intensity distribution as a function of the component of the neutron momentum transfer vector parallel to the sample's surface. This information can provide additional insight to extend the interpretation of the specular reflectivity measurements regarding in-plane correlations of the samples studied. [25] It is important to note that, since NR data are normalized to the incident neutron intensity, the measured SLD values are absolute and do not need to be arbitrarily scaled.

Differences in scattering length densities ( $S L D s)$ and the thickness of the various layers in the sample result in neutrons being reflected from the interfaces. The $S L D$ value, the product of the number density of atoms and their nuclear coherent scattering lengths, is unique for a particular chemical composition and specific structure of the measured film. Thus, it can be considered a fingerprint of the material. For a material of known stoichiometry and density, the SLD can be calculated according the Equation 2:

$$
S L D_{\text {neutron }}=\rho \frac{N_{A}}{M} \sum_{i=1}^{\text {atoms }} b_{i}
$$

where $b_{i}$ is the coherent scattering length of the $i^{\text {th }}$ atom, $\rho$ is the density of the material, $M$ is molecular mass and $N_{A}$ is Avogadro's number. In cases where the material is a mixture of phases with different stoichiometries Eq. 2 can be used to calculate the SLD by weighing the separate stoichiometric phases by their volume $\%$.

Interference of reflected neutrons results in the characteristic modulation of the intensity of the reflected beam, $R(Q)$. (See Fig. 1). The $R(Q)$ contains information about the $S L D$ distribution perpendicular to the sample surface, $S L D(z)$. Inferring $S L D(\mathrm{z})$ from $R(Q)$, however, is not trivial. Because the NR measurement only collects the intensity of the reflected beam and not its phase, there is no unique mathematical transformation from $R(Q)$ to $S L D(z)$. Therefore, NR data is usually interpreted by iteratively adjusting a trial $S L D(z)$ distribution until the reflectivity it predicts matches the measured reflectivity, $R(Q)$, to within a specified tolerance, $i$. e. lowest $\chi^{2}$ value in the least-squares refinement. The continuous function $S L D(z)$ may often be approximated by a series of discrete layers-referred to as "boxes" or "slabs" each with a constant SLD. Interlayer roughness may be taken into account using an error function centered at each interface or any other relevant functional form. Fitting the NR data provides information regarding the depth profile of the coherent SLD perpendicular to the sample surface with up to $\AA$ precision. To not over-parameterize the fitting of the NR data, the simplest, but physically meaningful, descriptions of the systems ( 2 and 3 slabs) were used. The typical $\chi^{2}$ values ranged from 8.6 to 36.9 .

\section{Computational Methods}

The lattice size, the density, and the SLD values of several hypothetical $\mathrm{ThO}_{\mathrm{y}}$ compounds were computed using hybrid density-functional theory (DFT). That was done because the NR data of the measured samples could not be modeled simply as a Th layer under a $\mathrm{ThO}_{2}$ layer. This suggested that nonstoichiometric thorium oxides layers might form in the process of thorium's oxidation. In the computations, the Th $6 s^{2} 6 p^{6} 5 f^{0} 6 d^{2} 7 s^{2}$ and $O 2 s^{2} 2 p^{4}$ electrons are explicitly treated as valence electrons.

The theoretical results are based on plane-wave expansions using the computer program VASP (Vienna Ab-initio Simulation Package). [26] The energy cut off for the plane-wave basis was set to 500 eV. Scalar relativistic effects are included with the PAW-PBE potentials available in the distributed code. [27] [28] The Brillouin zone was sampled by Monkhorst-Pack meshes of a $5 \times 5 \times 5$ grid for Heyd-Scuseria-Ernzerhof (HSE) hybrid functional calculations. [29] [30] The grid was tested at single points by expansion to a $6 \times 6 \times 6$ grid. No significant differences were found between them. All structural parameters (atomic position, and lattice constants) were relaxed using a conjugate-gradient algorithm until the Hellmann-Feynman forces were less than o.o1 eV/Å. Spin-orbit coupling has been implemented in VASP by Kresse and Lebacq. [26] The nonspherical contributions from the gradient corrections inside the PAW spheres were considered in the current calculations.

Hybrid functionals add a portion of the full, nonlocal, Hartree-Fock (HF) exchange to the standard local or semilocal exchange functional. The exchange-correlation energy then takes the form:

$E_{x c}=\alpha E_{x}^{H F}+(1-\alpha) E_{x}^{P B E}+E_{c}^{P B E},(\alpha=1 / 4)$

${ }_{\text {where }} E_{x}^{H F}$ is the full nonlocal HF exchange interaction, $E_{x}^{P B E}$ is a semilocal exchange interaction from the conventional 
PBE approximation to DFT, and $E_{c}^{P B E}$ is the corresponding PBE correlation energy. When $\alpha=1 / 4$, the approximation is known as $\mathrm{PBE}_{0}$. An important development for the application of these methods to solids was the development of "screened" hybrids. [28] Here, the concept of range separation is important: the interelectronic Coulomb potential is split into short-range and long-range components:

$$
\frac{1}{r_{12}}=S_{\omega}\left(r_{12}\right)+L_{\omega}\left(r_{12}\right)=\frac{\operatorname{erfc}\left(\omega r_{12}\right)}{r_{12}}+\frac{\operatorname{erf}\left(\omega r_{12}\right)}{r_{12}}
$$

where $r_{12}=\left|r_{1}-r_{2}\right|$, erf is the error function and governs long-range behavior, erfc is its short-range complement, and $\omega$ determines the screening length. That is, it is a parameter governing the extent of short-range interactions. The screened hybrid functional takes the form of the $\mathrm{PBE}_{\mathrm{o}}$ hybrid at short range and the PBE semilocal GGA at long range,

$$
\begin{aligned}
E_{x c}^{H S E}= & a E_{x}^{H F, S R}(\omega)+(1-a) E_{x}^{P B E, S R}(\omega) \\
& +E_{x}^{P B E, L R}(\omega)+E_{c}^{P B E},(\alpha=1 / 4)
\end{aligned}
$$

and can be viewed as an interpolation between these two limits. [29] [30] This functional has proven useful for studies of semiconductors and insulators, [31] particularly for the calculation of reasonable lattice constants and reliable band gaps when comparing with experimental data. [32] In our recent work, the band gap and electronic properties for actinide dioxides $\mathrm{AnO}_{2}(\mathrm{An}=\mathrm{Th}, \mathrm{Pa}, \mathrm{U}, \mathrm{Np}, \mathrm{Pu}$, and $\mathrm{Am})$ series [33] and $\mathrm{U}_{3} \mathrm{O}_{8}[34]$ were calculated.

The value for the empirical screening parameter, $\omega$, was chosen to reproduce PBE hybrid heats of formation in molecules
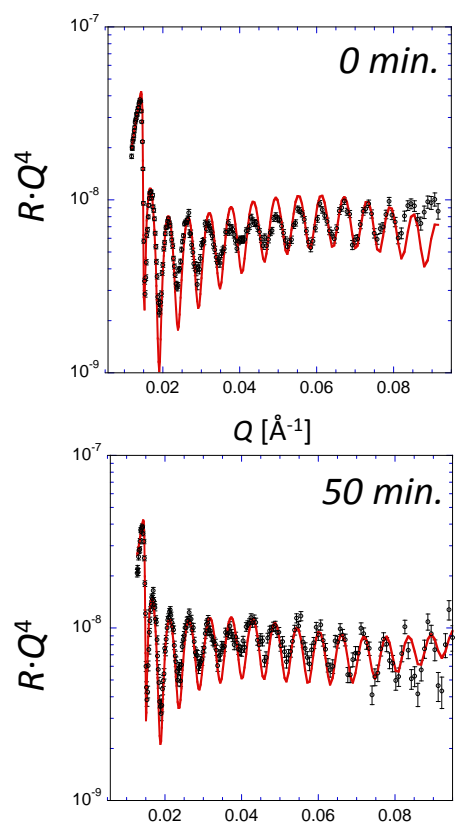

$Q\left[\AA^{-1}\right]$

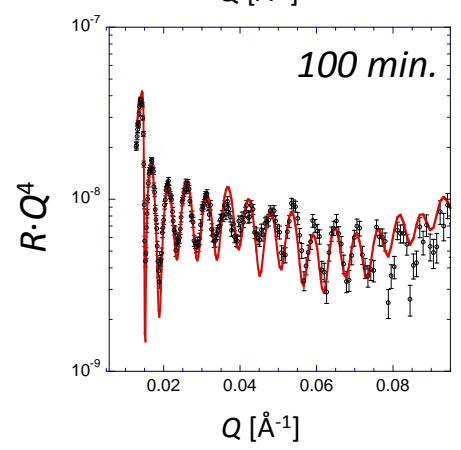

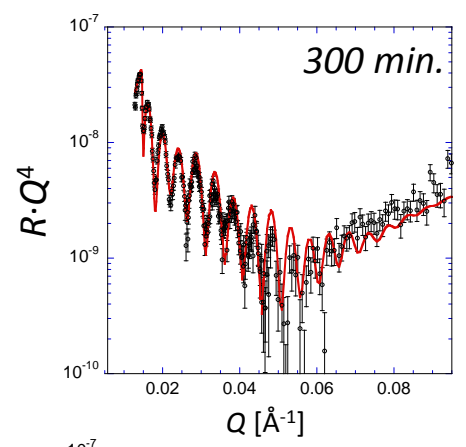

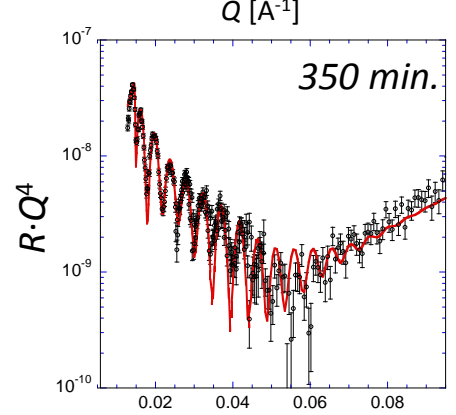

$Q\left[\AA^{-1}\right]$

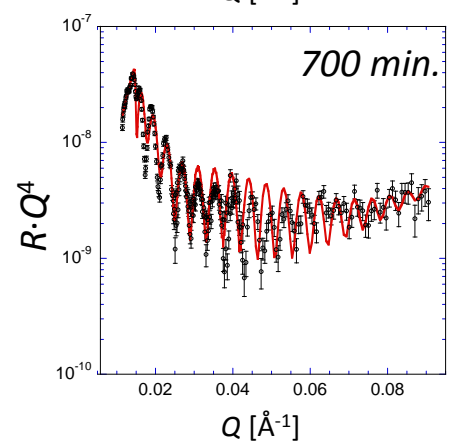
with some consideration given to band gaps in solids. Values in the range of approximately $0.2-0.3 \AA^{-1}$ all give similar quality results, corresponding to a screening length of $3-5 \AA$. In this work, $\omega$ is defined as $0.207 \AA^{-1}$ as originally suggested by Heyd et al. [30] There has been a previous report of the calculation of the structure and lattice-constants $B_{1}$ (rocksalt) ThO. [35] However, the properties of substoichiometric $\mathrm{ThO}_{\mathrm{w}}$ structures were not calculated nor the energetics of the movement of oxygen atoms through the lattice.

\section{Experimental Results and Discussion}

Fig. 1: Measured $R(Q) \cdot Q^{4}$ values (points with error bars) as a function of momentum transfer, $Q$, in units of reciprocal $\AA$ for increasing periods of exposure time to a $100 \mathrm{ppm}_{2}$ in argon mixture at $150^{\circ} \mathrm{C}$. The best-fit traces (solid lines) provide the SLD profiles shown in Fig. $2 \mathrm{a}$ and $b$. The term "best-fit" refers to a simplest real-space model with meaningful physical and chemical parameters yielding the lowest $\chi^{2}$ value in the least-squares refinement. Plotting reflectivity data $R(Q) \cdot Q^{4}$ versus $Q$ accounts for the $Q^{-4}$ falloff of the reflectivity data with increasing $Q$ due to the Fresnel law. It also allows for better visualization of the data and their fitting. 
The NR data of the sample under controlled oxidation conditions acquired at various exposure times are presented in Fig. 1. The calculated SLD profiles which provided the lowest chi-squared $\left(\chi^{2}\right)$ values in the least-squares refinement are shown in Fig. $2 \mathrm{a}$ and b. The $S L D$ profile of the sample before heating (Fig. 2a), termed run "0 min", showed the "as received" sample to be $\sim 1046$ $\AA$ thick. The top of the sample is seen to be a $\sim 44 \AA \mathrm{ThO}_{2}$ film. This superficial $\mathrm{ThO}_{2}$ is labelled (iii) in the figure. Thorium metal has an affinity for oxygen and water and, therefore, the formation of a thin native-oxide film is expected when the metal is
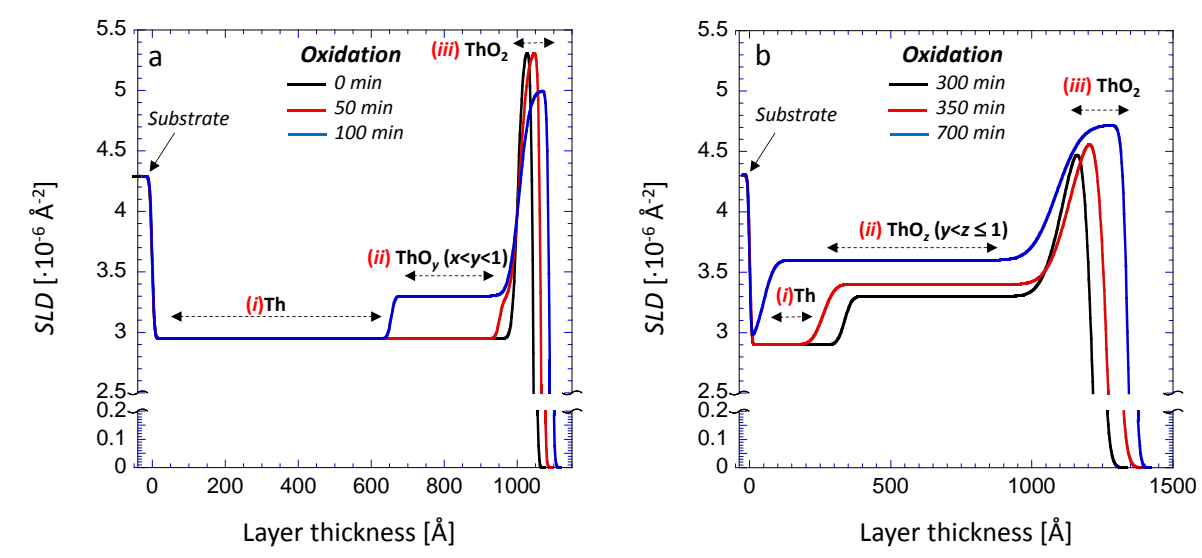

Fig. 2: SLD best-fit NR results of $\mathrm{Th} / \mathrm{ThO}_{\mathrm{y}}$ thin film as it is oxidized over time at approximately $150^{\circ} \mathrm{C}$. The zero thickness is set at the quartz substrate/Th film interface. $a$. The profile of the thin film before and during early stages of controlled oxidation (exposure to a $100 \mathrm{ppm} \mathrm{O}_{2}$ in argon mixture at $150^{\circ} \mathrm{C}$ ). $b$. Shows further reaction stages and long-term oxidation under the same conditions.

\begin{tabular}{|l|l|l|l|l|l|}
\hline & $\mathrm{O} \%$ & $\begin{array}{l}\text { Latt. } \\
\text { Const. }\end{array}$ & $\mathrm{V}\left(\AA^{3}\right)$ & $\begin{array}{l}\rho \\
\left(\mathrm{g} / \mathrm{cm}^{3}\right)\end{array}$ & $\begin{array}{l}\text { SLD }(\mathrm{x} \\
\left.10^{6} \AA^{-2}\right)\end{array}$ \\
\hline Th & & & & 11.7 & 3.13 \\
\hline Th4O & $20 \%$ & 5.294 & 148.38 & 10.57 & 3.17 \\
\hline Th2O & $33 \%$ & 5.394 & 156.92 & 10.16 & 3.37 \\
\hline Th4O3 & $43 \%$ & 5.410 & 158.34 & 10.04 & 3.63 \\
\hline ThO & $50 \%$ & 5.519 & 169.16 & 9.74 & 3.81 \\
\hline ThO2 & $67 \%$ & 5.586 & 174.25 & 10.07 & 5.03 \\
\hline
\end{tabular}

Table 1: Calculated values of the $S L D$ for Th and possible thorium oxide phases. The calculations of SLD are based on specific parameters (including the lattice constants) extracted from hybrid-density functional calculations. All the oxides are calculated to be FCC lattices with the oxygen occupying the octahedral positions. exposed to the atmosphere. In Fig. 2a $(i)$ marks the metallic thorium underneath the $\mathrm{ThO}_{2}$ overlayer.

Here are several observations prompted by the data plotted in the figures. During the oxidation process the total thickness of the film increased and a new layer formed between metallic Th and its $\mathrm{ThO}_{2}$ overlayer. The SLD of this layer below the $\mathrm{ThO}_{2}$ increased from the $3.3 \cdot 10^{-6} \AA^{-2}$ at $100 \mathrm{~min}$ to $3.6 \cdot 10^{-6} \AA^{-2}$ at 700 min. Even without modelling, one can see in Fig. 1 that the overall film thickness expanded substantially under the test conditions. There are 8.5 interference maxima before $\mathrm{Q}=0.060$ $\AA^{-1}$ for the sample at time 0 and 10 maxima at $300 \mathrm{~min}$. This amounts to approximately a $17 \%$ expansion of the overall film thickness. While there is surface and interfacial roughening, as the damping of interference fringes for high $Q$ data show, best fittings (Fig. 2) indicate only a modest increase of the $\mathrm{ThO}_{2}$ thickness to 106,138 and $257 \AA$ at 300,350 and 700 min., respectively. (These values are measured from the inflection points on either side of the thorium oxide peaks.) 
During the same interval, the new layer (ii) between the $\mathrm{ThO}_{2}$ and elemental Th dramatically expanded in thickness (see Fig. 2). We speculate that substoichiometric oxides $\left(\mathrm{ThO}_{\mathrm{x}, \mathrm{y}, \mathrm{z}}\right.$, where $\left.\mathrm{x}, \mathrm{y}, \mathrm{z} \leq 1\right)$ comprise this layer and that the metallic Th layer vanished under the $\mathrm{ThO}_{2}$ layers starting at the $\mathrm{ThO}_{2}$ interface. At the same time the SLD of this layer gradually increased suggesting that the oxygen content of the material was increasing. The SLD finally saturated (see $700 \mathrm{~min}$ ) with a composition labelled as $\mathrm{ThO}_{\mathrm{z}}$. The likely value of $z$ is 1 as discussed below.

DFT calculations provide some guidance what crystallographic phases of thorium oxides could be involved. Calculated SLD as well as crystallographic parameters for relevant $\mathrm{ThO}_{y}$ materials, with increasing $\mathrm{O} \%$, are presented in Table 1. First, it should be noted that, based on the SLD of the time $=0$ data, the sputtered Th layer is only $94 \%$ dense. The SLD calculated for the measured Th layer is 2.95 compared to the 3.13 of bulk Th. The presence of voids is consistent with what is expected for room-temperature, low-pressure sputter deposited thorium (melting point $=2087 \mathrm{~K}$ ) in structure-zone models. Thorium films sputter deposited under these conditions are expected to include voids associated with the grain boundaries. The SLD values given in the Table 1 are maximal values expected for each material.

The initial stages of low-temperature oxidation have been discussed by Fehlner. [28] In the Cabrera-Mott model for the growth of a protective oxide overlayer, electrons in a metal can tunnel $\sim 50 \AA$ A into an insulating layer. [29] The early stage of the oxidation of the thorium metal in this model begins by $\mathrm{O}_{2}$ molecules adsorption and proceeds by dissociation on the surface of the $\mathrm{ThO}_{2}$ layer to create reactive species. Both adsorption and dissociation depend on the microstructure and the electronic properties of this thin oxide film. Generally, with either an insulating or a semiconducting oxide surface film on the metal layer, the electrical and structural properties of the oxide film may be the controlling factors for the entire bulk metal oxidation process. For instance, the oxides may form dense O-impermeable layers which retard further rapid oxidation. In this case to pass through thicker layers, electrons must receive additional energy. Alternatively, a fractured oxide surface, or one with high grain-boundary density, may provide pathways for subsequent oxygen atoms or radicals to diffuse into the bulk of the metal. The NR results point to this latter alternative being the case here.

The NR data reveal that the $\mathrm{ThO}_{2}$ film does not protect the thorium metal from oxidation at $150^{\circ} \mathrm{C}$. This is shown in two ways. First, the thickness of the $\mathrm{ThO}_{2}$ increases linearly with time at a rate of $\sim 0.3 \AA /$ min, growing from $\sim 44 \AA$ thick at the beginning and reaching about $257 \AA$ at $700 \mathrm{~min}$. Second, the thorium under the $\mathrm{ThO}_{2}$ is rapidly converted along an advancing front to a suboxide, $\mathrm{ThO}_{w}$ (here, $w$ stands for all the subscripts $x, y, z$ indicating substoichiometric oxides). Specifically, after exposure to the oxidizing atmosphere at $150^{\circ} \mathrm{C}$ for $\sim 50 \mathrm{~min}$., an $\sim 59 \AA \mathrm{ThO}_{x}$ thick layer is formed, accompanied by a decrease of the thorium metal thickness. This new substoichiometric oxide layer, marked as (ii) in Fig. 2a and b, has a SLD value of $\sim 3.2 \times 10^{-6} \AA^{-2}$, which is larger than that of thorium metal and significantly lower than that of $\mathrm{ThO}_{2}$. After 100 minutes of oxidation, this new oxide layer grew to $\sim 351 \AA$ and its $S L D$ value increased to about $3.3 \times 10^{-6} \AA^{-2}$. The increase in the $S L D$ of this layer is interpreted as an increase in its effective oxygen content. For example, $\mathrm{Th}_{4} \mathrm{O}$ can become $\mathrm{Th}_{4} \mathrm{O}_{2}($ also written as $\left.\mathrm{Th}_{2} \mathrm{O}\right)$ and that, $\mathrm{Th}_{4} \mathrm{O}_{3}$. We do not know the exact composition of this region but denote it as $\mathrm{ThO}_{y}$, (where, $x$ $<y<1)$.

The structure of all the crystallographic phases presented in Table 1 for $w$ between 0 and 1 are calculated to be fcc with the oxygen atoms occupying the octahedral sites in the lattice forming rock-salt structure. Both Sun et al. [20] and our work suggest that ThO should have an fcc crystal lattice. Such compounds with early transition metals like titanium, zirconium, and hafnium are known to allow substoichiometry, so one might hypothesize that here the layer that forms has an $f c c$ crystal structure that starts with only partial filling of the available octahedral sites with $\mathrm{O}$ atoms, but that gradually, as more oxygen is available, the filling fraction tends to 1 . From time of $100 \mathrm{~min}$. to $300 \mathrm{~min}$., the suboxide region expands at rate of about $2.1 \AA / \mathrm{min}$. towards the substrate through the Th metal with an approximately constant SLD. This rate is significantly faster than the growth rate of $\mathrm{ThO}_{2}$.

Nonspecular scattered neutrons provide evidence that the oxidation proceeded homogenously towards the film's interior without creating significant surface roughness or in-plane islands. Measurements of nonspecular (or off-specular) scattered neutrons were recorded along with the specular signal. Presence of a significant number of off-specular neutrons would be a clear indication of the presence of in-plane inhomogeneities in the film created during the oxidation process, including development of surface or interfacial roughness. No significant off-specular signal was seen in the preliminary stages of the chemical evolution of the films.

The fitted $S L D$ profiles indicate increasing roughness between different layers (Fig. 2b), see also the Table S1 in Supplemental Materials. This can be seen in the NR spectra (Fig. 1) for $350 \mathrm{~min}$ which become plateau-like and the visibility of the interference fringes diminishes for $Q>0.06 \AA^{-1}$. The initial rms roughness parameters at 0 min. at the air/ThO ${ }_{2}, \mathrm{ThO}_{2}$ 
/Th, and Th/quartz interfaces were 6.1, 11.6 and $5 \AA$, respectively. At the final oxidation stage (700 min.) the roughness of the air/ $\mathrm{ThO}_{2}, \mathrm{ThO}_{2} / \mathrm{ThO}$ and $\mathrm{ThO} / \mathrm{Th}$ interfaces increased rather dramatically and were 17.5, 68.8 and $30 \AA$, respectively. However, due to the limited maximum $Q$ range $\left(\sim 0.085 \AA^{-1}\right)$ the roughness parameters are measured with rather limited certainties. At 350 min., an increase of both the thickness and SLD value, $\mathrm{ThO}_{z}(y \leq z \leq 1)$, is observed (region (ii) in Fig. 2b). Past this time, up to the experiment's end at $700 \mathrm{~min}$., the oxidation and disappearance of the thorium metal decreases significantly, and the SLD values of all three components asymptotically approach constants. In contrast to the decrease of the rate in the thorium metal oxidation after 350 min., the formation of $\mathrm{ThO}_{2}$ continues its constant slow growth rate (region iii in Fig. 2b).

A close inspection of the $S L D$ distribution obtained at $\sim 700 \mathrm{~min}$. shows $\sim 50 \AA$ of thorium metal remaining at the thorium metal/quartz substrate interface. This could indicate that this metallic thorium is isolated from the incoming oxygen or that the proximity with the $\alpha$-quartz substrate stabilizes it through heteroepitaxial growth when the film was deposited. In support of such a conjecture, it has been reported that $\mathrm{UO}_{2}$ thin films deposited on (100) $\mathrm{LaAlO}_{3}$ single crystal substrate are protected against oxidation up to $1000{ }^{\circ} \mathrm{C}$ when the $\mathrm{UO}_{2}$ grew epitaxially on the $\mathrm{LaAlO}_{3}$. [30] [31] [32]

One pieces of evidence that suggests that the phase labeled $\mathrm{ThO}_{z}$ is "ThO", that is, $z$ approaches 1 , is the observation that the SLD value of this phase saturates at $3.6 \times 10^{-6} \AA^{-2}$ for measurement made beyond $350 \mathrm{~min}$. Early on, the SLD of the layer rose gradually from as time increased, via the formation of a series of substoichiometric oxides, e.g. $\mathrm{ThO}_{x}, \mathrm{ThO}_{y}$ in can be deduced, but after 350 minutes the SLD value saturates. This suggests that the sample approaches a relatively stable, limiting, rock-salt ThO phase, at which time the maximum number of oxygen atoms/unit cell are incorporated into the lattice. Secondly, the SLD value calculated for this phase $\left(\sim 3.6 \times 10^{-6} \AA^{-2}\right)$ strongly suggests that the layer is indeed ThO with $\sim 5.5$ vol. \% of voids. $\left(\mathrm{Th}_{4} \mathrm{O}_{3}\right.$ would be possible only if it were $100 \%$ dense, which is not likely considering the extent to which the film expands.)

The hybrid density-functional, theoretical assessment discussed above permitted an investigation of the thermodynamic stability of $\mathrm{ThO}$ at ambient conditions. Table 2 provides the computed formation energy for $\mathrm{ThO}$ and $\mathrm{ThO}_{2}$. It is not surprising that the oxidation state of $\mathrm{Th}^{4+}\left(\mathrm{ThO}_{2}\right)$ is thermodynamically more stable than $\mathrm{Th}^{2+}(\mathrm{ThO})$. Being an early member of the actinide series, the energies of the $5 f$ and $6 d$ levels of thorium are nearly equal and are of the same order as chemical binding energies. In ThO, thorium is formally in a +2 -oxidation state and its $5 f$ orbitals are diffused enough to be involved in molecular bonding with the $s, d$ and $f$ orbitals with occupations of $1.84,0.71$, and 0.34 , respectively. [36] However, for $\mathrm{ThO}_{2}$, the outer $7 s$ electrons of thorium are gone, while the occupied and delocalized thorium $6 d$ and $5 f$ orbitals both contribute to the +4 bonding states with 1.27 and 0.69 occupancies, respectively.

More detailed investigations have been performed to consider the entire energy picture for $\mathrm{O}$ atoms to occupy different sites in the $f c c$ thorium lattice (including four octahedral sites and eight tetrahedral sites, respectively). Fig. 3 shows the comparison of the energy gains by incorporating $\mathrm{O}$ atoms into tetrahedral and octahedral sites in the $f c c$ thorium lattice. In the rock-salt $\mathrm{ThO}$ and $\mathrm{ThO}_{2}$ structures, all $\mathrm{O}$ atoms preferably occupy the octahedral and tetrahedral sites, respectively. One can see (Figure 3) that the octahedral sites are competitive with tetrahedral for accommodation of the first $\mathrm{O}$ atoms until the $\mathrm{O}$ occupancy is higher than $1(\mathrm{O}: \mathrm{Th}>1: 1)$. After that the fluorite structure, where $\mathrm{O}$ atoms occupy the tetrahedral sites, becomes more favorable. Accordingly, rock-salt $\mathrm{ThO}$ is not expected to be thermodynamically as stable as the $\mathrm{ThO}_{2} \mathrm{phase}$. However, whether the rock-salt structure is kinetically favored depends on the energy barriers for migrating $\mathrm{O}$ atoms along certain migration pathways (energy minima).

The energy barriers can be assessed by searching for the energy saddle point when an atom (i.e., $\mathrm{O}$ ) is added (oxidation) or removed (reduction). To study the energy barrier between an octahedral site and a tetrahedral site in the $f c c$ thorium lattice, the potential energy was calculated for various points along the pathway of the $\mathrm{O}$ migration between these sites, as shown in Fig. 4. The energy barrier when moving the $\mathrm{O}$ from octahedral to tetrahedral sites is around $0.55 \mathrm{eV}$ whereas it is around $0.25 \mathrm{eV}$ per atom for the other direction, respectively. It is therefore proposed that the $\mathrm{O}$ diffusion might be highly hindered, especially during the initial stages of the oxidation process. Consequently, the oxidation from $\mathrm{ThO}$ to $\mathrm{ThO}_{2}$ could be a relatively slow process even though the release of a large amount of heat is anticipated for the oxidation. 


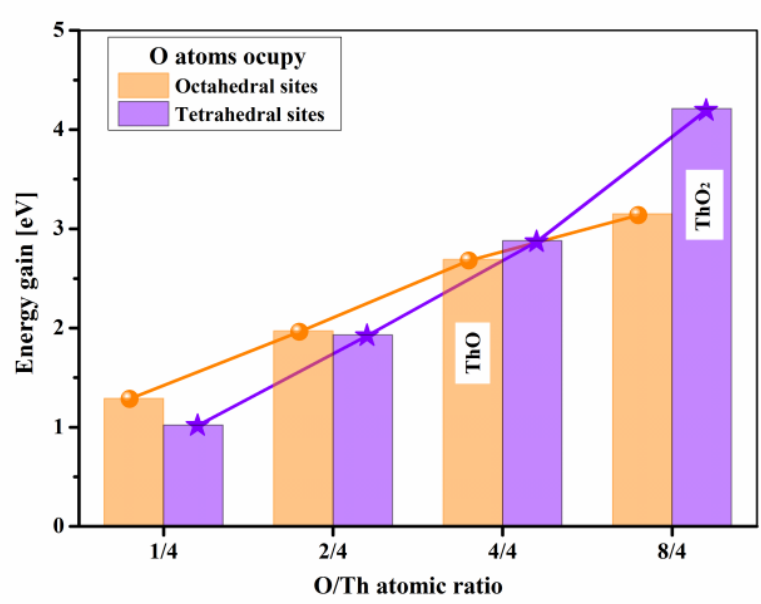

Fig. 3: Computed energy gain for different occupancy sites as a function of $\mathrm{O} / \mathrm{Th}$ ratio.

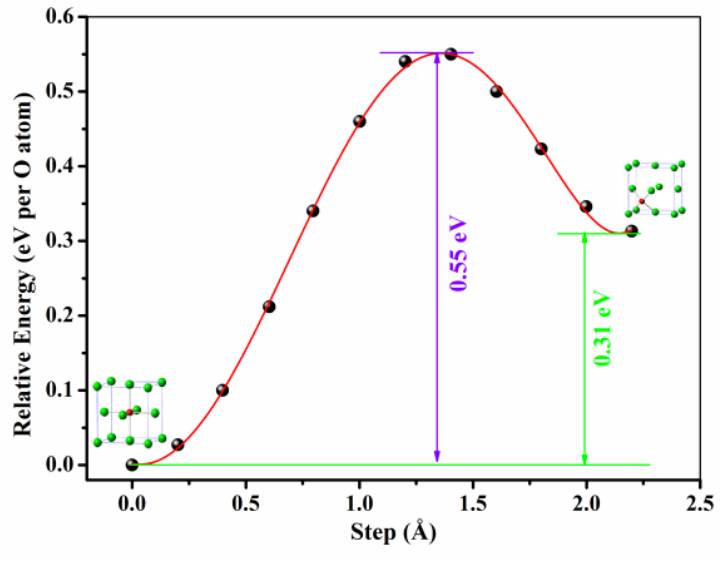

Fig. 4: Potential energy surface for the O diffusion from octahedral site to tetrahedral site.

In summary, time-resolved, in situ NR revealed several details about the oxidation of a thorium thin film under the conditions of low oxygen partial pressure $(100 \mathrm{pm})$ and moderate temperature $\left(150^{\circ} \mathrm{C}\right)$. First, the $\mathrm{ThO}_{2}$ native oxide was shown to be nonprotective. This is in contrast with bulk samples which previous studies have shown to be oxidation of thorium to be parabolic at this temperature and linear at higher temperatures. [37] [38] Oxidation to $\mathrm{ThO}_{2}$ proceeds from the outside towards the interior linearly and at a rate of $\sim 0.3 \AA / \mathrm{min}$. Second, a suboxide layer forms between unreacted thorium metal and its dioxide overlayer. The progress of this layer into the interior of the film is about 7 times as rapid as the formation of $\mathrm{ThO}_{2}$. The composition of the layer appears to pass through several discrete substoichiometric oxides, e.g. $\mathrm{ThO}_{x}$ and $\mathrm{ThO}_{y}$ (though it is difficult to identify the exact value of $x$ or $y$ from these measurements) and eventually approaches a relatively stable phase, $\mathrm{ThO}_{z}$. The SLD value measured for this phase $\left(\sim 3.6 \times 10^{-6} \AA^{-2}\right)$ and the layer thicknesses taken together, strongly suggests it is approximately stoichiometric ThO. Even though $\mathrm{ThO}_{2}$ is the thermodynamically more stable phase, NR data coupled with hybrid functional density calculations suggest that the metastable phase: ThO-is formed first and it is formed for kinetic reasons. Based on the computed formation energy it is seen that the oxidation of thorium is preferably begins with the incorporation of $\mathrm{O}$ atoms into the octahedral sites of the $f c c$ thorium lattice to form the rock-salt structure of substoichiometric oxides until the $\mathrm{Th} / \mathrm{O}$ ratio reach $1: 1$. Calculations indicate that the energy barrier for an $\mathrm{O}$ atom to migrate from an octahedral to a tetrahedral site is around $0.55 \mathrm{eV}$, while the energy barrier of opposite migration is around $0.25 \mathrm{eV}$ per atom, respectively, This suggests the incorporation of $\mathrm{O}$ atoms to form a fluorite structure of the dioxide is hindered, especially when the available $\mathrm{O}$ partial pressure to interact with the underneath Th metal is limited.

These results show the utility of time-resolved NR in understanding thin-film reactions that would be difficult to observe and quantify by any other technique alone. (Distinguishing between voids and oxygen content is straightforward by NR, but not by x-ray diffraction, for example.) A new metastable solid phase-ThO- has been revealed. The conditions which produce this phase for thin films may be useful in fabricating bulk materials, perhaps, beginning with submicron-sized thorium powders oxidized in a fluidized bed. Such inquiries merit further investigation.

\section{ASSOCIATED CONTENT}

None.

\section{AUTHOR INFORMATION}

\section{Corresponding Author}

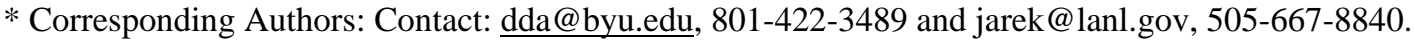

\section{Present Addresses}

$\dagger$ Current Address: Joint Research Laboratory for Nuclear Fuel Development, State Nuclear Power Research Institute, China

${ }^{*}$ Current Address: Intel Corp., Portland, OR., USA

${ }^{\S}$ Current Address: State Key Laboratory of Coal Conversion, Institute of Coal Chemistry, Chinese Academy of Sciences, Taiyuan, Shanxi 030001, China \& Synfuels China, Beijing 101407, China

\section{Author Contributions}

The manuscript was written through contributions of all authors. All authors have given approval to the final version of the manuscript. 


\section{ACKNOWLEDGMENT}

For financial support of this work, we gratefully acknowledge the support of the U.S. Department of Energy through the LANL/LDRD Program. This work benefited from the use of the time-of-flight neutron reflectometer (SPEAR) at the Lujan Neutron Scattering Center at LANSCE funded by the DOE Office of Basic Energy Sciences and Los Alamos National Laboratory under DOE Contract DE-AC52-06NA25396. We acknowledge the contributions of Michael Whitehead in the experimental setup.

\section{ABBREVIATIONS}

SLD, Scattering length density; NR, neutron reflectivity; ppm, parts per million; fcc, face-centered cubic; eV, electron-volt; $\chi^{2}$, chi-squared; TOF, time-of-flight; SPEAR, Surface Profile Analysis Reflectometer; VASP, Vienna Ab-initio Simulation Package; HSE, Heyd-Scuseria-Ernzerhof; DFT, Density Functional Theory.

\section{Bibliography}

[1] M. Avedesian, Microstructure of Magnesium and Magnesium Alloys, Materials Park, OH: ASM International , 1999.

[2] J. J. McKetta, Encyclopedia of Chemical Processing and Design: Thermoplastics to Trays, Separation, Useful Capacity, CRC Press, 1996.

[3] M. S. Wickleder, B. Fourest and P. K. Dorhourt, Thorium, Springer Science+Business Media, 2006.

[4] Hammond, C. R., The Elements, CRC Press, 2004.

[5] R. Benz, "Thorium-Thorium dioxide phase equilibria," Journal of Nuclear Materials, vol. 29, no. 1, pp. 43-49, 1969.

[6] J. Emsley, Nature's Building Blocks, Oxford University Press, 2001.

[7] S. R. Daly, "Synthesis and Properties of a Fifteen-Coordinate Complex: The Thorium Aminodiboranate," Angewandte Chemie-International Edition, vol. 49, pp. 3379-3381, 2010.

[8] International Atomic Energy Agency, Thorium fuel cycle — Potential benefits and challenges, VIENNA, A-1400: IAEA, 2005, pp. 1-131.

[9] World Nuclear Association, "Thorium," World Nuclear Association, September 2015. [Online]. Available: http://www.world-nuclear.org/information-library/current-and-futuregeneration/thorium.aspx. [Accessed 17 November 2016].

[10] E. Shwageraus, P. Hejzlar and M. Kazimi, "Use of Thorium for Transmutation of Plutonium and Minor Actinides in PWRs," in Seventh Information Exchange Meeting on Actinide and Fission Product Partitioning and Transmutation, Jeju, Republic of Korea, 2002.

[11] V. Sobolev and S. Lemehov, "Modelling Heat Capacity, Thermal Conductivity of Dioxide Thermal Expansion, and Components of Inert Matrix Fuel," Journal of Nuclear Materials, vol. 352, pp. 300$308,2006$.

[12] S. Krishnamurty, "The band systems of thorium oxide and hafnium oxide," Proceedings of the Physical Society of London Section A, vol. 64, no. 381, p. 852, 12 July 1951.

[13] A. J. Darnell, W. A. McCollum and T. A. Milne, "Vapor Pressure of Thorium," The Journal of Physical Chemistry, vol. 64, pp. 341-346., 1960. 
[14] C. T. Dewberry, K. C. Etchison and S. A. Cooke, "The Pure Rotational Spectrum of the ActinideContaining Compound Thorium Monoxide," Physical Chemistry Chemical Physics, vol. 9, p. 4895, 2007.

[15] B. Liang, L. Andrews, J. Li and B. E. Bursten, "Experimental and Theoretical Studies of the Products of Laser-Ablated Thorium Atom Reactions with $\mathrm{H} 2 \mathrm{O}$ in Excess Argon," Journal of the American Chemical Society, vol. 124, pp. 6723-6733, 2002.

[16] L. Andrews, Y. Gong, B. Liang, V. E. Jackson, R. Flamerich, S. Li and D. A. Dixon, "Matrix Infrared Spectra and Theoretical Studies of Thorium Oxide Species: ThOx, and Th2Oy," Journal of Physical Chemistry A, vol. 115, pp. 14407-14416, 2011.

[17] M. C. Heaven, "Probing Actinide Electronic Structure Using Fluorescence and Multi-Photon Ionization Spectroscopy," Physical Chemistry Chemical Physics, vol. 8, pp. 4497-4509, 2006.

[18] F. Wang, A. Le, T. C. Steimle and M. C. Heaven, "Communication: The Permanent Electric Dipole Moment of Thorium Monoxide," The Journal of Chemical Physics, p. 134, 2011.

[19] L. Skripnikov and A. V. Titov, "Theoretical study of thorium monoxide for the electron electric dipole moment search: Electronic properties of $\mathrm{H} 3 \Delta 1$ in ThO," J. Chem. Phys. , vol. 142, p. $024301,2015$.

[20] W. W. Sun, W. Luo and R. Ahuja, "Stability of a new cubic monoxide of Thorium under pressure," Scientific Reports, vol. 5, pp. 1-5, 4 September 2015.

[21] R. J. Ackermann and E. G. Rauh, "The Preparation and Characterization of the Metastable Monoxides of Thorium and Uranium," Journal of Inorganic and Nuclear Chemistry, vol. 35, pp. 3787-3794, 1973.

[22] R. S. Searcy and A. W. Newbury, "The Composition and Properties of the Solid Produced by Reaction of Thorium with Hydrochloric Acid," Inorganic Chemistry, vol. 1, no. 4, pp. 794-798, 1962.

[23] H. He, P. Wang, D. D. Allred, J. Majewski, M. P. Wilkerson and K. D. Rector, "Characterization of Chemical Speciation in Ultrathin Uranium Oxide Layered Films," Analytical Chemistry, vol. 84, p. 10380-10387, 2012.

[24] M. Dubey, P. Wang, M. S. Jablin, M. Mocko and J. Majewski, "SPEAR-ToF Neutron Reflectometer at the Los Alamos Neutron Science Center," Eur. Phys. J. Plus, vol. 126, pp. 7-11, 2011.

[25] M. Jablin, M. Zhernenkov, B. Toperverg, M. Dubey, H. Smith, A. Vidyasagar, R. Toomey, A. Hurd and J. Majewski, "In-plane Correlations in a Polymer-supported Lipid Membrane Measured by off-specular Neutron Scattering," Phys. Rev. Letters, vol. 106, p. 138101, 2011.

[26] G. Kresse and J. Hafner, "Ab Initio Molecular Dynamics for Liquid Metals," Physical Review B, vol. 47, pp. 558-561, 1993.

[27] P. E. Blöchl, "Projector Augmented-Wave Method," Physical Review B, vol. 50, pp. 17953-17979, 1994.

[28] G. Kresse and D. Joubert, "From Ultrasoft Pseudopotentials to the Projector Augmented-Wave 
Method," Physical Review B, vol. 59, pp. 1758-1775, 1999.

[29] J. Heyd, G. E. Scuseria and M. Ernzerhof, "Hybrid Functionals Based on a Screened Coulomb Potential," The Journal of Chemical Physics, vol. 118, pp. 8207-8215, 2003.

[30] J. Heyd, G. E. Scuseria and M. E. Ernzerhof, "Hybrid Functionals Based on a Screened Coulomb Potential [J. Chem. Phys.118, 8207 (2003)]," The Journal of Chemical Physics, vol. 124, p. 219906, 2006.

[31] T. M. Henderson, J. Paier and G. E. Scuseria, "Accurate Treatment of Solids with the HSE Screened Hybrid," physica status solidi (b), vol. 248, pp. 767-774, 2011.

[32] J. Heyd, J. E. Peralta, G. E. Scuseria and R. L. Martin, "Energy Band Gaps and Lattice Parameters Evaluated with the Heyd-Scuseria-Ernzerhof Screened Hybrid Functional," The Journal of Chemical Physics, vol. 123, pp. 174101-8, 2005.

[33] X.-D. Wen, R. L. Martin, L. E. Roy, G. E. Scuseria, S. P. Rudin, E. R. Batista, T. M. McCleskey, B. L. Scott, E. Bauer, J. J. Joyce and T. Durakiewicz, "The Effect of Spin-Orbit Coupling on the Actinide Dioxides AnO2 ( $\mathrm{An}=\mathrm{Th}, \mathrm{Pa}, \mathrm{U}, \mathrm{Np}, \mathrm{Pu}$, and $\mathrm{Am}$ ): A Screened Hybrid Density Functional Study," The Journal of Chemical Physics, vol. 137, pp. 154707-6, 2012.

[34] X.-D. Wen, R. L. Martin, G. E. Scuseria, S. P. Rudin, E. R. Batista and A. K. Burrell, "Screened Hybrid and DFT + U Studies of the Structural, Electronic, and Optical Properties of U3O8," Journal of Physics: Condensed Matter, vol. 25, p. 025501, 2013.

[35] I. R. Shein, K. I. Shein and A. L. Ivanovskii, "First-principle study of B1-like thorium carbide, nitride and oxide," Journal of Nuclear Materials, vol. 353, p. 19-26, 2006.

[36] X. Wang, L. Andrews, K. S. Thanthiriwatte and D. A. Dixon, Inorg. Chem., vol. 52, pp. 10275$10285,2013$.

[37] A. Gerds and M. Mallett, "Surface Reaction between Oxygen and Thorium," The Journal of the Electrochemical Society, vol. 101, no. 4, pp. 171-174, 1954.

[38] P. Levesque and D. Cubicciotti, "The Reaction between Oxygen and Thorium," J of the Amer. Chem. Soc., vol. 73, pp. 2028-31, 1951.

[39] F. P. Fehlner, Low-Temperature Oxidation: Role of Vitreous Oxides, John Wiley and Sons, Inc., 1986.

[40] H. He, D. A. Andersson, D. D. Allred and K. D. Rector, "Determination of the Insulation Gap of Uranium Oxides by Spectroscopic Ellipsometry and Density Functional Theory," The Journal of Physical Chemistry C, vol. 117, pp. 16540-16551, 2013.

[41] N. F. Mott, "The Theory of the Formation of Protective Oxide Films on Metals, Ii.," Transactions of the Faraday Society, vol. 35, pp. 472-483, 1940.

[42] A. K. Burrell, T. M. McCleskey, P. Shukla, H. Wang, T. Durakiewicz, D. P. Moore, C. G. Olson, J. J. Joyce and Q. Jia, "Controlling Oxidation States in Uranium Oxides through Epitaxial Stabilization," Advanced Materials, vol. 19, pp. 3559-3563, 2007.

[43] R. Jilek, "Preparation of Epitaxial Uranium Dicarbide Thin Films by Polymer-Assisted 
Deposition," Chemistry of Materials, vol. 25, pp. 4373-4377, 2013.

\section{Figure Captions:}

Fig. 1: Measured $R(Q) \cdot Q^{4}$ values (points with error bars) as a function of momentum transfer, $Q$, in units of reciprocal $\AA$ for increasing periods of exposure time to a $100 \mathrm{ppm} \mathrm{O}_{2}$ in argon mixture at $150^{\circ} \mathrm{C}$. The best-fit traces (solid lines) provide the SLD profiles shown in Fig. 2a and b. The term "bestfit" refers to a simplest real-space model with meaningful physical and chemical parameters yielding the lowest $\chi^{2}$ value in the least-squares refinement. Plotting reflectivity data $R(Q) \cdot Q^{4}$ versus $Q$ accounts for the $Q^{-4}$ falloff of the reflectivity data with increasing $Q$ due to the Fresnel law. It also allows for better visualization of the data and their fitting.

Fig. 2: SLD best-fit results of $\mathrm{Th} / \mathrm{ThO}_{\mathrm{y}}$ thin film under oxidation over time obtained from NR at approximately $150^{\circ} \mathrm{C}$. The zero thickness is set at the quartz substrate/Th film interface. $a$. The profile of the thin film before and during early stages of controlled oxidation (exposure to a $100 \mathrm{ppm} \mathrm{O}_{2}$ in argon mixture at $150^{\circ} \mathrm{C}$ ). $b$. Shows further reaction stages and longtime oxidation under the same conditions.

Fig. 3: Computed energy gain for different occupancy sites as a function of $\mathrm{O} / \mathrm{Th}$ ratio.

Fig. 4: A cut through the potential energy surface along path of least energy for the $\mathrm{O}$ diffusion from octahedral site to tetrahedral site. 

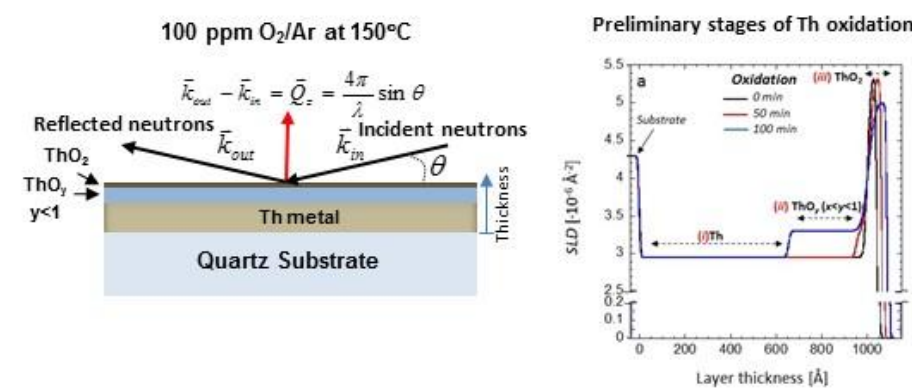